\title{
V-A was The Key
}

\author{
Steven Weinberg \\ Department of Physics, The University of Texas at Austin \\ weinberg@physics.utexas.edu
}

My scientific interaction with George Sudarshan goes back almost half a century to 1958. I was a new post-doc at Columbia University, when I got a call from Bob Marshak, who was the head of the physics department at the University of Rochester, inviting me to come up to Rochester for a week or two to work with him and George Sudarshan and two other people. One of these two I am glad to see here today - Susumu Okubo. Okubo is famous, but the other one, Werner Teutsch, sadly died before he made a name for himself. The invitation was to come up to Rochester and work with them on the currents of weak interactions. I was absolutely thrilled by this invitation for two reasons. One reason was that I was a brand new post-doc, and this was the first time that anyone had ever invited me to go anywhere and pay my plane fare. The other reason for being excited was that, at that time, the whole world of physics was excited by the work that Marshak and Sudarshan had done. They were trailing clouds of glory, because of their development of the $V-A$ theory of weak interactions. This was a great opportunity to work with them.

We wrote two papers together, and I don't think I am going to hurt George's feelings if I say that these were not the most important papers that either of us wrote. They were of some importance, but not great. To me the important thing was getting to know George and Bob Marshak and Okubo, and participating in the intellectual excitement over the breakthrough in the theory of weak interactions.

At that time I did not understand how important that breakthrough would prove to be. It opened up the door to a line of theoretical work, which in less than a decade led to a thorough understanding of things that had earlier, when I was a graduate student, seemed like a mélange of miscellaneous facts that had no coherence and certainly no beautiful theoretical interpretations. What I'd like to do is first go backward in time from 1958 and remind you of some of the prehistory of this and then from 1958 go forward and describe the developments that followed from the $V-A$ theory.

I suppose it really starts from Enrico Fermi's great paper on the theory of beta decay in 1933 , a paper, incidentally, that was rejected by Nature magazine when it was submitted on the grounds that it was purely mathematical and not really physical. What Fermi did was to initiate the theory of weak interactions. Weak interactions, and in particular the weak interaction of nuclear beta decay, were supposed to occur because of a term in the Hamiltonian of the world involving the product of four fields - electron, neutrino, proton, and neutron. Another way one can think of this is that an electron and neutrino together would interact with a current operator that, unlike the electromagnetic current, does not leave the charge of the nucleus unchanged, but actually converts a neutron into a proton or vice versa. Because of the possibility of an analogy with electromagnetism, Fermi originally thought of this as a vector current, like the electric current. It

1

Published under licence in Journal of Physics: Conference Series by IOP Publishing Ltd. 
soon became clear on experimental grounds that this could not be the whole story. Fermi's theory beautifully accounted for the spectrum shape of beta decay (that is the distribution of electron energies between the maximum and minimum) in the fastest beta decays, the so-called allowed beta decays; beta decays that we later understood were decays in which the electron and neutrino receive no orbital angular momentum from the decay process. These allowed beta decays have just the spectrum shape that Fermi's theory predicted, but Fermi's theory also led to selection rules that simply were violated in nature. With a purely vector current, in allowed beta decay - where there's no orbital angular momentum given to the electron and neutrino - the nucleus cannot change its spin. This created no problem at all with, say, the decay of a neutron into a proton, both of which have spin $1 / 2$. That process was allowed. But there were other known beta decays, which Fermi's theory would forbid. One example is the decay of the ground state of Boron-12 or Nitrogen-12 into the ground state of Carbon-12. While the ground states of Boron-12 and Nitrogen-12 both have spin 1, the ground state of Carbon-12 has spin 0, so that decay would be forbidden with just a vector current. And yet, those were discovered to be perfectly good allowed beta decays, going as fast as you would expect of decays in which no orbital angular momentum was exchanged. So it was clear that something more than vector currents was needed.

I think it was Belinfante who first showed that the most general possibility, again, assuming that no orbital angular momentum is transferred in the fundamental decay process, was a mixture of five types of current: scalar, vector, tensor, axial vector, and pseudoscalar. This may be unfamiliar to today's graduate students in physics, because the work of Marshak and Sudarshan made this kind of theoretical classification obsolete. We now know what the right answer is, so who studies these other possibilities anymore? But, I find history fascinating, so allow me here to look back on this bit of history.

It was clear that you had to have scalar or vector, because the other currents would not produce transitions between nuclear states of 0 spin, and there are transitions like the decay of Oxygen-14 into Nitrogen-14 where both nuclei have spin 0 . That is allowed by the scalar and vector current interactions, but would not be allowed by the other interactions. Then there are transitions like the one I mentioned when Boron-12 and Nitrogen-12 decay into the ground state of Carbon-12, which require the presence of tensor or axial vector currents. So you have scalar and/or vector plus tensor and/or axial vector currents - in some mixture that no one knew. (The pseudoscalar interaction doesn't produce allowed beta decay, so it is a somewhat tangential possibility.)

And this is where beta decay theory stayed for a long time. There were experiments on forbidden beta decays where angular momentum is transferred, not in the fundamental decay process but through more complicated nuclear physics - these are highly suppressed beta decays - which show that you couldn't have scalar and axial vector, and you couldn't have tensor and vector. And then there were experiments that showed you couldn't have scalar and vector, or tensor and axial vector. After a long history, it appeared that the interaction had to be scalar and tensor or vector and axial vector. The experimental evidence was for a while overwhelming in favor of scalar and tensor, which would quite rule out any analogy with electromagnetism, whose current is a vector current.

As I recall, there were a number of experiments that indicated scalar and tensor, and every once in a while one of those experiments was shown to be wrong. But then another experiment was done which restored the status quo, and again showed scalar and tensor. (I'm talking from my memory, rather than from serious historical research.) By the late 1950s, this picture was beginning to fray at the edges and, in particular, was inconsistent with the idea of universality. I think the thing that most stood out was the problem of the decay of the pi-meson. You know, the main decay of the pi-meson is into a muon and a neutrino. (I'm not being careful in this talk to distinguish neutrinos from anti-neutrinos, but you all know which it is in these decays.) But, a pion can occasionally decay instead into an electron and a neutrino. Now the ratio of those two 
processes - if you assume the universal interaction, that is if you assume that electrons and muons, and their associated neutrinos (in those days we didn't know that there were associated neutrinos - we thought there was only one type of neutrino - but today we can say 'and their associated neutrinos') interact in exactly the same way, as they seem to be in all respects identical except for their mass, then as Ruderman and Finkelstein showed, you can very neatly predict the ratio of the decay rates. There are only two possibilities for the currents in this decay that are allowed by Lorentz invariance: axial vector and pseudoscalar. Since, at that time most people thought the fundamental interaction was scalar and tensor, and with pseudoscalar being allowed but unconstrained by nuclear beta decay, the natural assumption was that in pion decay the interaction is pseudoscalar rather than axial vector, since there didn't seem to be any axial vector in beta decay. Well, that would predict that the pion would decay overwhelmingly into electrons rather than into muons, because since electrons are lighter, there's more energy available for the decay. But this was in complete contradiction with the observed fact that the pion almost always decays into a muon, not an electron, plus a neutrino.

On the other hand, if the current was axial vector, then because of the peculiar fact that in that case the decay amplitude would be proportional to the mass of the muon or the electron, and since the muon is 210 times heavier than the electron, the decay rate would be almost 210 squared times greater for pion decay into muons than into electrons, which was what was observed.

The whole issue was clouded by the fact that for a long time experimenters couldn't find the electron mode at all. Since the electron made had a rate that apparently was even below the theoretical expectation for axial vector decay, many physicists assumed that for some reason the rate must be zero. (I lost a dollar to Norman Kroll betting on that.) But it was also clearly a possibility that the experimenters had just missed the electron mode, and that in fact the current in pion decay was pure axial vector. But that contradicted everything that was known from beta decays.

So, as Jan Nilsson said here, it took tremendous courage for Marshak and Sudarshan to propose, in order to save universality, and on the grounds of other experiments who perhaps other speakers will discuss, that in fact the weak interaction was vector and axial vector, and not, as had been thought, scalar and tensor. And, of course, subsequent experiment showed that they were right.

This may all sound like a technicality: scalar, vector, tensor, axial vector, pseudoscalar -- who really cares? Fermi didn't even think it necessary to explore these other possibilities, and then experimentalists had beaten away on this problem for decades, and now we had vector and axial vector. So what?

Well, it turned out that this really was the key to the future. The analogy with electromagnetism was restored in the first place, the analogy that originally motivated Fermi. It wasn't long before Feynman and Gell-Mann suggested that to a good approximation the vector current in beta decay is actually conserved, in the same sense that the electromagnetic current was conserved. That is that the rate of change of its time component plus the divergence of its space component equals zero. Gell-Mann discussed various experiments that would test this, and very soon these experiments showed that the idea was right. Because of their suggestion of the conservation of current, Feynman and Gell-Mann (who had independently suggested the $V-A$ theory) I think received more than their share of credit for the $V-A$ theory. I believe the first public statement of the $V-A$ theory was in a paper by Sudarshan and Marshak presented by George in a conference at Padua. (If my memory of that is wrong, doubtless others will correct me). But, perhaps because of their suggestion of a conserved vector current, or perhaps because Feynman and Gell-Mann were already justly famous for other work, and George, after all, was just beginning his career, people tended to refer to this as the Feynman and Gell-Mann Theory. They don't now. I think that with the passage of time, the history has gotten sorted out and it's now generally realized that the earliest statement of the $V-A$ theory was due to Marshak and Sudarshan. Marshak was very upset about this, and I remember a series of international 
conferences where some speaker would stupidly refer to the Feynman Gell-Mann Theory and Marshak would get up and complain. George was much more of a gentleman and would never publicly complain, and left it to the judgment of history, which has finally been rendered with fairness on this point.

Now, the vector current was known to be conserved. The axial vector current rather obviously couldn't be conserved. In fact, there were obvious objections to that idea. Axial vector current conservation would imply effects in beta decay that were grossly in contradiction to observation. Nevertheless, Yoichiro Nambu saw through this problem and realized that if the pion mass were zero, then the exchange of a pion between the initial and final nucleus and the leptons - the muon or electron and the neutrino - would provide a canceling current which would allow the axial vector current to be conserved without any violation of experimental knowledge.

So this we had a kind of peculiar situation, that in a mythical world with a zero pion mass, the axial vector current would be conserved. This led to a number of interesting predictions. Right away, this allowed an explanation of the success of the calculation of Goldberger and Treiman of the rate of pion decay, which had seemed mysterious because the approximations made by Goldberger and Treiman were difficult to understand. But, with Nambu's idea of an approximately conserved axial vector current, which would be exactly conserved if the pion were massless, you could actually derive the Goldberger-Treiman formula as an exact formula for the pion decay amplitude in the limit when the pion mass is zero. The pion mass, of course, is not zero, but the pion is by far the lightest of all the strongly interacting particles. So, as an approximation, this didn't seem outrageous.

But this was still a peculiar situation. After all, the electromagnetic current was exactly conserved, and this continues to be true to this day. No one challenges it. But the vector current was not exactly conserved, as Feynman and Gell-Mann had recognized, because neutrons and protons don't have exactly the same mass. And the axial vector current is even less exactly conserved. It couldn't be conserved if the pion mass were not zero, and it isn't.

So what's going on here? There was a lot of concentration at this time on the nature of these currents, as my discussion shows. But it was gradually realized that instead of concentrating so much on the properties of the currents themselves, we should concentrate instead on the symmetries of the strong interactions. After all, electromagnetic current conservation is just a manifestation of the fact that there is a symmetry governing electromagnetic phenomena known as electromagnetic gauge invariance. The conserved vector current of Feynman and Gell-Mann is approximately conserved because of the approximate symmetry of strong interactions known as the isotopic spin symmetry. The approximately conserved axial vector current is conserved -- in that weird sense that Nambu suggested -- because of an approximate symmetry of the strong interaction known as chiral symmetry. But this symmetry is not apparent in formulas for ratios of masses or decay rates. If it were, then for instance the nucleon mass would have to be zero. Instead chiral symmetry is spontaneously broken. That is, though it is a symmetry of the underlying equations, it is not a symmetry of the solution of these equations, the particles and their interactions. Goldstone first suggested that whenever you have a spontaneously broken symmetry, you get a massless particle - a so called Goldstone boson, sometimes called a NambuGoldstone boson - of which the pion is an archetype. (This was proved as an apparently quite general theorem by Goldstone, Salam, and me.) The pion would be massless if the axial vector symmetry were exact.

There was a lot of confusion at the time about the relation between approximate symmetry and broken symmetry, because many people thought they had something to do with each other. I remember as late as 1975 , Heisenberg was suggesting that isotopic symmetry was really an exact symmetry, and only appeared to be an approximate symmetry because it was spontaneously broken. This is really nonsense, because in general when a symmetry is broken, it does not appear as an approximate symmetry - it is completely gone and is manifested not in symmetry 
relationships like ratios of amplitudes, but instead in theorems about interactions of the Goldstone bosons. It took a while for this understanding to sort itself out.

My own work in the mid 1960's was on the theory of spontaneously broken symmetry developing it not from the point of view of weak interaction currents but from the point of view of spontaneously breaking an approximate symmetry of strong interactions - the chiral symmetry. But of course, that work, which led to all sorts of soft pion theorems and so on, could not have been done without the realization that there was a partially conserved axial vector current, which could not have been done without the first realization that beta decay proceeds through vector and axial vector currents.

For a while the theorem of Goldstone, Salam, and me - that every time you have a spontaneously broken symmetry, you get a massless Goldstone boson - was regarded as an obstacle to the further development of spontaneous symmetry breaking in particle physics. Because you don't need a giant accelerator to produce massless particles. Especially if the particles have strong interactions, they should be pouring out of experiments at just about any accelerator. Since this doesn't happen, one could easily rule out the existence of new massless particles. In fact, when Salam, Goldstone, and I wrote our paper on this, I contributed an epigraph, a line from King Lear: "nothing can come from nothing," expressing our sense of disappointment with the failure of the idea that approximate symmetries could be explained through the spontaneous symmetry breaking of exact symmetries, or in other words, by the lack of symmetry in the vacuum.

Well, it turned out that spontaneous symmetry breaking did have a future ahead of it, and not only in the theory of low-energy pions. There was an exception to the theorem of Goldstone, Salam, and me, found about the same time by Higgs, by Brout and Englert, and by Guralnik, Hagen, and Kibble, That exception was (as already realized by Anderson in the theory of superconductivity) that if the symmetry is a local symmetry (a gauge symmetry) then instead of the Goldstone boson appearing as a massless particle, it instead appears as a zero helicity state of a massive vector particle, so you would not have any of these unwanted massless Goldstone bosons. Salam and I independently both seized on this as something that would provide an opportunity for a theory of weak and electromagnetic interactions based on an exact but spontaneously broken symmetry. Instead of Goldstone bosons, which should have been observed, you have massive vector particles which transmit the weak and electromagnetic interactions.

That idea of massive vector particles was by no means new. Indeed this is another way in which the V-A theory was the key. As long as you had scalar and tensor currents in the weak interactions, there would be no possibility of weak interactions being transmitted by anything like a photon - a vector field. As soon as the V-A theory came along, many theorists - I remember papers by T. D. Lee and others --- Schwinger, Glashow --- started exploring the possibility that the weak interactions were transmitted by a field which interacts both in a parity conserving and a parity non-conserving way to produce both a vector and an axial vector coupling of beta decay. This idea of intermediate vector bosons was ubiquitous. Everyone knew that an intermediate vector boson had to be heavy because it had not been seen. (Generally speaking, when people said "heavy," they meant ten times the proton mass or something like that.) Experimenters started searching for intermediate vector bosons in cosmic rays and accelerators.

Several theorists showed that having a massive vector boson would lead to mathematical problems, precisely because of their helicity zero component. Spin-1 massless particles have only helicity \pm 1 states but a spin- 1 massive particle has helicity \pm 1 and 0 states. That zero helicity state of the spin-1 massive particle leads to terrible problems - in particular ultraviolet divergences that could not be removed by renormalization theory. When Salam and I, in the late 60's, proposed our theory of weak and electromagnetic interactions based on the exact but spontaneously broken symmetry, we hoped - and both of us expressed it in our respective papers - that because the local symmetry in the theory was exact, the theory would avoid the problems that were present with a massive vector particle. I attempted unsuccessfully to prove it. The proof was finally 
presented in 1971 by a young graduate student, Gerardus 't Hooft. That finally convinced at least the theoretical community of particle physicists that there was something to this theory.

Those were great days and I am enjoying reminiscing about them. But none of this - not the development of spontaneous symmetry breaking ideas, not the idea of a conserved vector current, and not the unified theory of weak and electromagnetic interactions which is based on spontaneous symmetry breaking ideas - none of that could have occurred without the key breakthrough of the realization that there really is a profound analogy between weak and electromagnetic interactions. It is not that the weak interactions are produced only by a vector current, as Fermi had thought; it certainly is not that weak interactions are produced by scalar and tensor interactions which had dominated the thinking of nuclear physicists for so many years; rather, weak interactions are produced by both vector and axial vector currents, as with great courage and physical insight and élan, Marshak and Sudarshan were the first to propose in 1957.

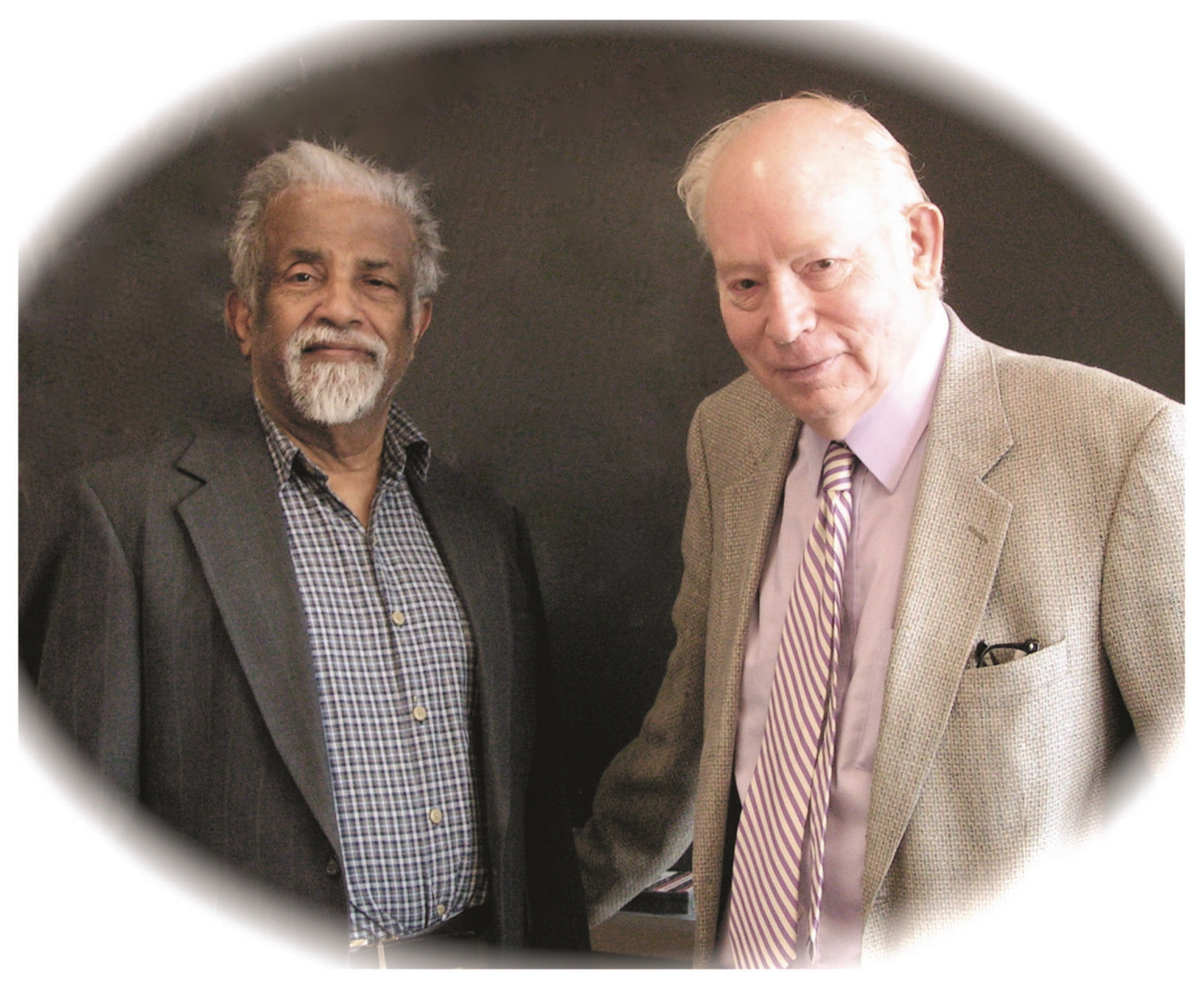

